\title{
Manejo atual das metástases hepáticas de câncer colorretal - recomendações do Clube do Fígado de São Paulo
}

\author{
Current management of liver metastases from colorectal cancer - \\ recommendations of the São Paulo Liver Club
}

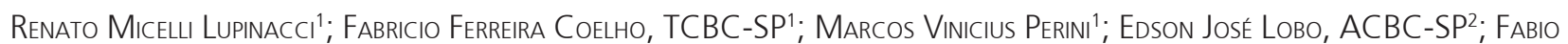

Gonçalves Ferreira, TCBC-SP3; Luiz Arnaldo Szutan, TCBC-SP3; Gaspar de Jesus lopes, TCBC-SP2; Paulo Herman, TCBC-SP1

\section{R E S U M O}

\begin{abstract}
Aproximadamente metade dos pacientes portadores de câncer colorretal apresenta metástases hepáticas durante a evolução de sua doença que afetam diretamente o prognóstico e são diretamente responsáveis por $2 / 3$ dos óbitos relacionados à doença. Nas últimas duas décadas o tratamento das metástases hepáticas de câncer colorretal (MHCCR) proporcionou ganho expressivo na sobrevida quando todas as opções terapêuticas são colocadas à disposição do paciente. Nesse contexto, o tratamento cirúrgico persiste como a única possibilidade de cura com índices de sobrevida em cinco anos de 25 a $58 \%$. No entanto, apenas $1 / 4$ dos pacientes tem doença ressecável ao diagnóstico. Por essa razão, um dos pontos fundamentais no manejo atual dos pacientes com MHCCR é o desenvolvimento de estratégias que possibilitem a ressecção completa das lesões hepáticas. O advento e aperfeiçoamento dos métodos ablativos expandiram as possibilidades da terapêutica cirúrgica, além disto, o surgimento de novos esquemas quimioterápicos e a introdução das terapias-alvo proporcionou altas taxas de resposta e alteraram definitivamente o manejo destes pacientes. O tratamento multimodal e a utilização da experiência de diversas especialidades médicas permitiram que o tratamento das MHCCR se aproximasse cada vez mais do tratamento ideal, ou seja, individualizado. Baseado em uma extensa revisão da literatura e na experiência de alguns dos centros especializados mais importantes do Brasil, o Clube do Fígado de São Paulo iniciou um trabalho de discussão multi-institucional que resultou nas recomendações que se seguem. Essas recomendações, no entanto, não visam ser absolutas, mas sim ferramentas úteis no processo de decisão terapêutica desse grupo complexo de pacientes.
\end{abstract}

Descritores: Neoplasias. Neoplasias hepáticas. Neoplasias colorretais. Metástase neoplásica. Terapêutica.

\section{INTRODUÇÃO}

O câncer colorretal (CCR) ocupa a terceira posição nas estatísticas mundiais de incidência de doenças malignas, com aproximadamente 1.200 .000 casos por ano, atrás apenas do câncer de pulmão e mama. Nos países desenvolvidos, o CCR já ocupa o primeiro lugar dentre as causas de neoplasia, quando analisados ambos os sexos ${ }^{1}$. Nos Estados Unidos, em 2010, são estimados mais de 142.000 casos e mais de 51.000 óbitos relacionados à doença ${ }^{2}$. O número de casos novos estimados para esta afecção no Brasil, em 2010, é de aproximadamente 28.000 casos, sendo o quarto câncer mais frequente nos homens e o terceiro em mulheres ${ }^{3}$.
O sítio mais comum de metástases do carcinoma colorretal é o fígado, sendo acometido em até 75\% dos pacientes que desenvolvem alguma metástase desta afecção. Em 15 a 25\% dos pacientes, detectam-se metástases hepáticas já ao diagnóstico do tumor primário, as quais são denominadas sincrônicas ${ }^{4}$. Além disso, 12 a $40 \%$ dos pacientes desenvolverão doença metastática hepática após a ressecção do tumor primário, chamada de metacrônica, a maioria nos primeiros três anos de evolução da doença ${ }^{4}$. Desta forma, cerca de metade dos pacientes portadores de CCR têm ou terão metástase hepática durante a vida, sendo que 20 a 50\% destes apresentarão doença metastática somente no fígado.

Demais membros do Clube do Fígado.

Adriano Miziara Gonzalez-TCBC-SP2; Marcelo Moura Linhares-TCBC-SP2; Suzan Menasce Goldman²; Alberto Goldenberg-TCBC-SP2; Tarcisio Trivino-TCBC-SP2; Mauricio Alves Ribeiro,ACBC-SP3; Paulo Celso B. Massarollo-TCBC-SP33, Jaime A. Pirola Kruger-ACBC-SP; Vincenzo Pugliese$\mathrm{ACBC}-\mathrm{SP} 1$

1. Faculdade de Medicina da Universidade de São Paulo; 2. Universidade Federal de São Paulo - Escola Paulista de Medicina; 3. Faculdade de Ciências Médicas da Santa Casa de São Paulo. (Faculdade de Ciências Médicas da Santa Casa de São Paulo; Faculdade de Medicina da Universidade de São Paulo; Universidade Federal de São Paulo - Escola Paulista de Medicina). 
As metástases hepáticas afetam diretamente o prognóstico, sendo responsável por ao menos 2/3 dos óbitos relacionados à doença ${ }^{5}$. Ao avaliarmos a história natural de pacientes com metástases hepáticas de câncer colorretal (MHCCR) potencialmente ressecáveis, mas que se opuseram ao tratamento cirúrgico, encontramos sobrevida média de 14 a 24 meses, sendo esta virtualmente nula em cinco anos ${ }^{6}$.

Por outro lado, o CCR mesmo com doença à distância, é uma das neoplasias que apresentou maior benefício com relação ao ganho de sobrevida quando tratada adequadamente. Por essa razão, passou a ser uma das patologias mais estudadas na literatura, havendo na última década grande incremento nos conhecimentos referentes aos fatores de risco, prognósticos, biologia e genética tumorais, o que determinou uma nova compreensão da doença e a possibilidade de desenvolvimento de novas e efetivas modalidades terapêuticas.

O tratamento cirúrgico é o único que comprovadamente propicia a possibilidade de cura para os pacientes portadores de MHCCR, com índices de sobrevida em cinco anos de 25 a $58 \%$ e em dez, de 21 a $29 \%{ }^{5,7}$. No entanto, apenas 20 a $25 \%$ das metástases hepáticas são potencialmente ressecáveis ao diagnóstico e, portanto, potencialmente curáveis ${ }^{5,6}$.

Por essa razão, um dos pontos fundamentais no manejo atual das MHCCR é o desenvolvimento de estratégias que possibilitem a ressecção completa (macro e microscópica) das lesões hepáticas. Nesse contexto, o desenvolvimento anestésico e técnico permitiu a realização de ressecções hepáticas maiores com segurança, a embolização portal e o advento dos métodos ablativos expandiram as possibilidades da terapêutica cirúrgica, o que, associado ao surgimento de novos esquemas quimioterápicos, com altas taxas de resposta clínica, alteraram definitivamente as bases do manejo destes pacientes.

Os esquemas mais modernos de quimioterapia utilizando 5-fluoroacil associado à oxaliplatina (FLOX/ FOLFOX) ou irinotecan (FOLFIRI) apresentam taxas de resposta acima de $50 \%$, auxiliando no controle perioperatório da doença em pacientes com neoplasia ressecável ou diminuindo o volume tumoral de modo a tornar possível a ressecção em 10 a 40\% dos pacientes inicialmente considerados com doença irressecável ${ }^{8,9}$. A possibilidade recente de utilização de novos agentes biológicos antiangiogênicos como o cetuximab ou o bevacizumab pode incrementar ainda mais esses resultados, com respostas objetivas acima de $60 \%{ }^{10}$.

Atualmente, a terapêutica ideal e que garante os melhores resultados é multimodal e deve ser individualizada para a situação clínica de cada paciente, sendo imprescindível a participação em conjunto dos diversos profissionais envolvidos, tais como o radiologista, o radiologista intervencionista, o oncologista e o cirurgião. Estudos recentes apontam para os bons resultados quando se utiliza quimioterapia perioperatória associada à ressecção cirúrgica completa das lesões hepáticas, com sobrevida atuarial em cinco anos de 51 a $64 \%$, sendo $70 \%$ destes sem recidiva da doença ${ }^{11}$

Baseado nas melhores evidências disponíveis na literatura e na experiência de alguns dos centros especializados mais importantes do país, o Clube do Fígado de São Paulo iniciou um trabalho de discussão multiinstitucional para a geração de recomendações quanto ao manejo terapêutico das MHCCR. Essas recomendações, no entanto, não visam ser absolutas, mas sim ferramentas úteis no embasamento de decisões clínicas concernentes ao tratamento desse grupo complexo de pacientes.

\section{DIAGNOSTICO E ESTADIAMENTO}

O diagnóstico das MHCCR pode ser estabelecido durante o estadiamento pré-operatório, ato operatório ou durante os exames de rotina realizados no acompanhamento pós-operatório de pacientes submetidos à ressecção do CCR. A maioria dos serviços utiliza para o diagnóstico precoce das MHCCR a dosagem periódica do antígeno carcinoembrionário (CEA), em geral a cada três meses, associada a exame de imagem abdominal (ultrassonografia, ou, preferencialmente, tomografia computadorizada ou ressonância magnética) numa frequência mínima anual, por pelo menos três anos. Novos exames podem ser realizados, principalmente quando houver aumento do CEA ou sinais e sintomas de alerta. Apesar de menos consensual, é recomendável ainda a realização concomitante de exames para avaliação torácica (tomografia ou radiografia de tórax), pelo risco de metástases pulmonares silenciosas ${ }^{12}$.

O CEA não tem valor diagnóstico para o CCR, mas valor prognóstico. A determinação do valor basal e o seu seguimento no pós-operatório podem chamar a atenção quando há aumento em seus níveis séricos para o aparecimento de doença secundária, em especial hepática ${ }^{12}$, com sensibilidade de $64 \%$ e especificidade de $90 \%{ }^{13}$. Eleva-se principalmente na presença de MHCCR de maior volume, porém para pequenos nódulos, pode não cursar com elevação significativa. Dados sobre o marcador CA199 no diagnóstico e seguimento das MHCCR ainda são controversos e seu uso não está estabelecido.

O diagnóstico das MHCCR é quase sempre realizado através de exames de imagem abdominais, tais como ultrassonografia ou exames contrastados de tomografia computadorizada ou ressonância magnética. Todos os métodos são efetivos, havendo acurácia semelhante para lesões maiores, no entanto, para lesões pequenas, a tomografia e ressonância apresentam melhor sensibilidade e especificidade. Para lesões menores do que $1 \mathrm{~cm}$ pode haver benefício na utilização da ressonância magnética com contraste superparamagnético ${ }^{14}$. 
O estadiamento dos pacientes com MHCCR completa-se com a avaliação do segmento torácico. Recomenda-se a realização de tomografia computadorizada de tórax (que pode ser feita sem o emprego de contraste endovenoso), pois estes pacientes têm alta taxa de nódulos pulmonares subcentimétricos (cerca de $40 \%$ ), sendo que até $1 / 3$ destes são diagnosticados como metástases pulmonares no seguimento ${ }^{15}$. Pela raridade, demais exames para avaliação de outros sítios de metástases (cerebrais ou ósseas) não são realizados rotineiramente, mas somente na presença de sintomas clínicos que o justifiquem.

Atualmente, inúmeros trabalhos têm destacado a possibilidade da utilização do mapeamento de corpo inteiro através de exame radioisotópico com tomografia com emissão de pósitrons (PET, do inglês "Positron emission tomography") com glicose marcada com flúor-18 (fluorodeoxiglicose - FDG). Essa técnica conhecida como PET-SCAN baseia-se na ávida captação da glicose marcada pelas células do $\mathrm{CCR}^{16}$. Também foi relatada para este propósito a utilização de método que combina no mesmo exame a avaliação funcional dos órgãos ou tecidos obtida pelo PET-SCAN com as imagens estruturais obtidas pela tomografia (PET-CT) com resultados animadores ${ }^{16}$.

O emprego do PET-SCAN ou PET-TC visa selecionar os melhores candidatos à ressecção cirúrgica. As evidências existentes apontam para um aumento da acurácia diagnóstica das metástases, em especial as extra-hepáticas quando comparada aos métodos radiológicos habituais. Dentre as limitações do método destacam-se a diminuição da sensibilidade e valor preditivo positivo em lesões pequenas, em especial menores do que $1 \mathrm{~cm}$, lesões com histologia mucinosa e após quimioterapia ${ }^{17}$. Estudos indicam que estes podem alterar a conduta terapêutica em até $25 \%$ dos pacientes, evitando laparotomias ou procedimentos desnecessários ${ }^{18}$. Existe, entretanto, controvérsia se essa a seleção dos pacientes obtida implica em ganho na sobrevida global em longo prazo.

Atualmente, não existe embasamento científico para o uso sistemático do PET-SCAN ou PET-CT no algoritmo de estadiamento de pacientes portadores de MHCCR. Em grande parte dos pacientes essas modalidades não trarão informações adicionais para o manejo clínico, além disso, o custo e a acessibilidade restrita também são fatores limitantes. O papel desses novos métodos ainda está sendo avaliado, mas, em algumas situações clínicas, seu uso pode trazer benefícios. Dentre estas podemos citar pacientes com alto risco (CEA elevado) sem evidência de doença aos métodos convencionais, lesão hepática com características duvidosas à tomografia ou ressonância, suspeita de doença metastática extrahepática ou suspeita de recidiva do tumor primário. O contexto clínico em que o PET-SCAN ou PET-CT pode oferecer maior auxílio é naquele em que a descoberta de novas lesões intra ou extra-hepáticas pode contraindicar a terapêutica cirúrgica ${ }^{18}$
Mesmo com os modernos métodos diagnósticos o ultrassom intraoperatório (USIO) é fundamental no estadiamento das MHCCR e não deve ser prescindido, já que pode descobrir novas lesões e mudar a conduta cirúrgica em 11 a $30 \%$ dos casos $^{19}$.

Após o tratamento adequado das MHCCR, cerca de 50 a $70 \%$ dos pacientes apresentarão recidiva da doença no pós-operatório ${ }^{20}$. O principal local de recorrência é o próprio fígado, sendo, em 20 a 30\% dos casos, o único sítio de recidiva ${ }^{20}$. Desta forma, deve-se realizar o seguimento intensivo destes pacientes após a ressecção hepática, com exames de imagem e CEA a cada três ou quatro meses nos primeiros dois anos e a cada seis meses nos anos seguintes, até completar cinco anos de acompanhamento 21 .

Recomendações: O seguimento dos pacientes operados por CCR deve ser feito com a dosagem de CEA a cada três meses, associada a exames de imagem abdominal (ultrassonografia, ou, preferencialmente, exames contrastados de tomografia ou ressonância). Após o diagnóstico, o estadiamento de pacientes portadores de MHCCR deve ser completado com tomografia de tórax. Exames de imagem para avaliação de outros sítios de metástases (como as cerebrais ou ósseas) não devem ser realizados rotineiramente, mas somente na presença de sintomas clínicos que justifiquem essa conduta. O PET-SCAN ou PET-CT deve ser utilizado seletivamente, principalmente em pacientes com lesões hepáticas com características duvidosas à tomografia ou ressonância, suspeita de doença metastática extra-hepática ou suspeita de recidiva do tumor primário. O USIO deve ser realizado em todos os casos, pois, mesmo com os modernos métodos, pode alterar a conduta cirúrgica em percentual significativo de casos. Como os pacientes submetidos à ressecção de MHCCR têm grande chance de recidiva tumoral, em especial hepática, deve-se acompanhá-los com exames de imagem e dosagem de CEA a cada três ou quatro meses nos primeiros dois anos e a cada seis meses nos anos seguintes, até completar cinco anos de acompanhamento.

\section{DEFINIÇÃO DE RESSECABILIDADE}

Pode-se dividir os pacientes com MHCCR em portadores de doença inicialmente ressecável, doença potencialmente ressecável e aqueles com doença considerada irressecável. Uma vez completado o estadiamento, a ressecabilidade das lesões metastáticas hepáticas sincrônicas é de aproximadamente 10\% e das metacrônicas de 20 a $25 \%{ }^{6}$.

A definição de irressecabilidade, no entanto, pode ser bastante complexa, pois um grande número de variáveis deve ser analisado. Os parâmetros utilizados são: o número e tamanho dos nódulos, localização, possibilidade de obtenção de margem cirúrgica oncológica adequada, doença multinodular e/ou bilateral e evidência de doença 
extra-hepática (controlável x não controlável). Adicionalmente, a experiência do cirurgião tem impacto fundamental no resultado do tratamento. Idealmente, as ressecções hepáticas devem ser realizadas em centros especializados devido à alta complexidade do procedimento. Considerase como centro especializado, aquele que além de um grande número de ressecções hepáticas realizadas, apresenta índices de mortalidade para este procedimento inferior a 3 a $5 \% 22$.

A definição de ressecabilidade mais aceita pela maioria dos centros para o tratamento cirúrgico das MHCCR é a possibilidade de exérese total das lesões (macro e microscópica) e preservação de pelo menos 20 a $25 \%$ de parênquima (em fígados não doentes) com efetiva drenagem biliar, vascularização e drenagem venosa. Margens maiores do que $1 \mathrm{~cm}$ são recomendadas, mas não obrigatórias, sendo necessária a presença de margens microscopicamente livres ${ }^{23}$. Da mesma forma, a presença de doença extra-hepática ressecável também não impede o tratamento cirúrgico das lesões hepáticas ${ }^{5}$. As principais contraindicações à hepatectomia em portadores de MHCCR são: impossibilidade de controle do tumor primário; presença de tumor extra-hepático não ressecável, incluindo doença linfonodal ou peritoneal disseminada; multinodularidade impedindo a ressecção total das lesões com preservação de volume adequado de parênquima funcional; e lesões próximas ou invadindo grandes vasos de modo que impeçam tecnicamente a ressecção ou comprometam a vascularização do remanescente hepático ${ }^{24}$.

\section{MHCCR METACRONICAS}

A definição de MHCCR metacrônica é bastante variável na literatura. Existem autores que chamam de metacrônicas quaisquer metástases diagnosticadas após o tumor primário, outros classificam as metástases desta forma quando são diagnosticadas três meses, seis meses ou um ano após a ressecção do tumor primário. Na literatura, as metástases metacrônicas são diagnosticadas em 12 a $40 \%$ dos pacientes com CCR, a maioria nos primeiros três anos de evolução da doença ${ }^{4}$.

Estudos indicam que as MHCCR metacrônicas têm um melhor comportamento biológico e prognóstico mais favorável quando comparadas às sincrônicas, tendendo a serem menos numerosas, menos frequentemente bilobares e com valor de CEA mais baixo. Com relação aos resultados em longo prazo, os pacientes portadores de MHCCR metacrônicas adequadamente tratadas tem sobrevida em cinco anos 8 a $16 \%$ maior que as sincrônicas ${ }^{25}$.

Essas peculiaridades determinam diferenças fundamentais no manejo terapêutico deste subgrupo de pacientes. Para discussão, dividiremos os portadores de MHCCR metacrônicas em dois grandes grupos: os com doença ressecável e aqueles com doença irressecável, incluindo neste os pacientes com doença potencialmente ressecável.

\section{a) Doença ressecável}

No algoritmo de tratamento das MHCCR metacrônicas consideradas ressecáveis existem dois caminhos possíveis: a ressecção cirúrgica imediata ou a utilização de tratamento neoadjuvante (antes do procedimento cirúrgico). Ambos devem associar-se com realização de quimioterapia no período pós-operatório (quimioterapia adjuvante).

Classicamente, a conduta inicial adotada é a cirúrgica, com ressecção completa macro e microscópica do tumor. Nos últimos anos, no entanto, tem-se acumulado evidências de que o tratamento neoadjuvante pode trazer benefícios no controle da doença ressecável. As vantagens apontadas para o uso da quimioterapia neoadjuvante são aumento das taxas de exérese completa do tumor, diminuição do volume hepático a ser ressecado e possibilidade de ablação de eventuais micrometástases, além da avaliação da biologia tumoral, identificando os casos de maior gravidade ${ }^{24}$. A ausência de resposta à quimioterapia é um fator consistentemente associado a um pior prognóstico, com diminuição da sobrevida em longo prazo. Desta forma, o emprego da quimioterapia neoadjuvante poderia selecionar os pacientes com prognóstico mais favorável e, assim, os melhores candidatos à terapia cirúrgica ${ }^{26}$.

As potenciais vantagens da quimioterapia neoadjuvante, entretanto, ainda não demonstraram influenciar significativamente a sobrevida global dos pacientes em longo prazo ${ }^{26,27}$. Em estudo prospectivo randomizado, Nordlinger et al. ${ }^{28}$, comparando esquema de tratamento com quimioterapia perioperatória com FOLFOX vs. cirurgia isolada, mostraram potencial benefício no uso da quimioterapia neoadjuvante em pacientes com doença inicialmente ressecável. Demonstraram tendência a ganho de sobrevida livre de doença em três anos (35,4\% vs. $28,1 \%)$ sem, no entanto, atingir significância estatística numa análise de intenção de tratamento. Já no subgrupo de pacientes que foram efetivamente operados, observouse aumento da sobrevida livre de doença em três anos de $9,2 \%$. Outros estudos também apontam para ganho de sobrevida livre de doença em pacientes com MHCCR ressecáveis submetidos à quimioterapia neoadjuvante ${ }^{29}$

A utilização da quimioterapia, por outro lado, não é desprovida de complicações. Alguns estudos não encontraram aumento significativo da morbimortalidade relacionada à quimioterapia neoadjuvante, outros autores, no entanto, demonstraram aumento da das taxas de morbidade no período pós-operatório ${ }^{30}$. O próprio trabalho de Nordlinger et al. ${ }^{28}$, evidenciou aumento da taxa de complicações pós-operatórias no subgrupo de pacientes submetidos à neoadjuvância ( $25 \%$ vs. $16 \%$ ).

Os efeitos adversos da quimioterapia relacionamse com a toxicidade das drogas usualmente utilizadas e com o número de ciclos realizados. Karoui et al. ${ }^{30}$., mos- 
traram que pacientes submetidos a mais de seis ciclos antes da cirurgia tem aumento da morbidade pós-operatória. Da mesma forma, Aloia et al. ${ }^{31}$ demonstraram aumento da taxa de reoperação e tempo de internação em pacientes submetidos a 12 ou mais ciclos de 5 -fluoracil e FOLFOX como terapia neoadjuvante. Estabeleceu-se, então, na maioria dos serviços, que nos pacientes com MHCCR inicialmente ressecáveis deve-se empregar no máximo seis ciclos (aproximadamente três meses) de quimioterapia neoadjuvante, o que seria tempo suficiente para avaliar a resposta e evitar a toxicidade de drogas quimioterápicas ${ }^{30,31}$

A esteatose hepática é reportada em 30 a 47\% dos pacientes em uso de 5 -fluoracil ${ }^{32}$. O irinotecan pode induzir esteatohepatite (CASH, do inglês "chemotherapyassociated steatohepatitis") inclusive com desenvolvimento de fibrose e risco de disfunção hepática pós-operatória. O uso da oxaliplatina, por sua vez, está associado à dilatação e congestão sinusoidais e, do ponto de vista macroscópico, o fígado ganha coloração azulada ("blue liver"), havendo correlação com maior risco de sangramento intraoperatório.

Como a maioria das lesões induzidas pelos quimioterápicos relaciona-se com o número de ciclos e é reversível, regredindo em algumas semanas, pontos importantes na estratégia de utilização de tratamento neoadjuvante em lesões ressecáveis são: definição do tempo ideal de quimioterapia e do tempo entre a sua suspensão e o procedimento cirúrgico. A resposta radiológica pósquimioterapia pode ser avaliada após três ou quatro ciclos $(1,5 \text { a dois meses })^{33}$. Menores taxas de complicação foram demonstradas quando se opera o paciente após quatro a cinco semanas do término do ciclo de quimioterapia. Assim, recomenda-se realizar a ressecção cirúrgica, especialmente as maiores, após esse período para que não haja acréscimo na morbimortalidade pós-operatória ${ }^{34}$.

No caso da utilização de agentes antiangiogênicos, como bevacizumab (anticorpo monoclonal contra o fator de crescimento endotelial VEGF), o tempo entre a interrupção do medicamento e a cirurgia deve ser de seis a oito semanas pelo risco de sangramento, diminuição da regeneração hepática e dificuldades de cicatrização ${ }^{35}$.

A quimioterapia adjuvante mostrou benefício no tratamento de pacientes com MHCCR ressecáveis. Estudos controlados demonstraram benefício do uso desta no pós-operatório de pacientes submetidos à ressecção hepática por MHCCR em termos de sobrevida livre de doença e sobrevida global em cinco anos ${ }^{11}$. Em meta-análise recente, Wieser et al. ${ }^{36}$ demonstraram ganho de sobrevida livre de doença com quimioterapia sistêmica perioperatória nesse subgrupo de pacientes.

As recomendações atuais para o tratamento das MHCCR metacrônicas ressecáveis devem levar em conta a possibilidade de ressecção total das lesões, os avanços da quimioterapia e, em especial, os fatores prognósticos. Dessa forma, a cirurgia pode ser realizada inicialmente em pacientes com baixo risco clínico e sem fatores de mau prognóstico, deixando-se a quimioterapia para o pós-operatório (quimioterapia adjuvante). Os candidatos a essa conduta, em geral, tem nódulo metacrônico único ou poucos nódulos, de tamanho pequeno (até $5 \mathrm{~cm}$ ) localizados em posição favorável para ressecção ${ }^{28,37}$. Nos demais pacientes, principalmente naqueles com metástases ressecáveis, mas com fatores de mau prognóstico, como por exemplo, múltiplos nódulos, tamanho acima de $5 \mathrm{~cm}$, linfonodos positivos no tumor primário e altos níveis de CEA, recomenda-se a realização da quimioterapia perioperatória (neo e adjuvante) associada à ressecção cirúrgica completa das lesões ${ }^{24}$.

Com relação ao procedimento cirúrgico, este deve seguir os preceitos oncológicos e funcionais já apresentados: ressecção completa das lesões com margens microscopicamente livres deixando pelo menos 20 a $25 \%$ do parênquima hepático. Apesar de recomendáveis, margens maiores que $1 \mathrm{~cm}$ não são necessárias; margens subcentimétricas, porém microscopicamente livres, garantem resultados tardios semelhantes às margens mais

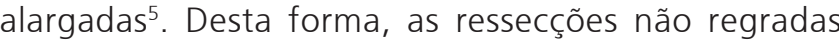
(enucleações), visando preservar parênquima hepático, têm espaço no tratamento da MHCCR. Os resultados não demonstram aumento da morbidade operatória, maior taxa de recidiva tumoral ou pior sobrevida em longo prazo quando comparados com pacientes submetidos à ressecções segmentares anatômicas ${ }^{38}$

Recomendações: O tratamento das MHCCR metacrônicas ressecáveis pode ser realizado pela ressecção cirúrgica imediata ou utilizando-se quimioterapia neoadjuvante. Independente da estratégia escolhida, o cirurgião deve buscar sempre a ressecção completa macro e microscópica do tumor. A quimioterapia pós-operatória (adjuvante) deve ser associada em todos os casos. Recomendamos a utilização de esquema quimioterápico neoadjuvante para a grande maioria dos pacientes pelos seus potenciais benefícios. O tempo de quimioterapia preconizado para evitar suas complicações é de três ou quatro ciclos (aproximadamente 1,5 a dois meses), momento em que a avaliação radiológica da resposta tumoral deve ser realizada. Após o término da quimioterapia deve-se esperar quatro semanas para realizar a hepatectomia. No caso da utilização de agentes antiangiogênicos, como bevacizumab, o tempo entre a interrupção do medicamento e a cirurgia deve ser de seis a oito semanas. Reserva-se a terapêutica cirúrgica inicial principalmente para os pacientes com baixo risco clínico e sem fatores de mau prognóstico, sendo os principais candidatos a essa conduta, os portadores de nódulo metacrônico único ou poucos nódulos, de tamanho pequeno (até $5 \mathrm{~cm}$ ) localizados em posição favorável para a ressecção.

\section{b) Doença irressecável}

Corresponde ao principal grupo de pacientes portadores de MHCCR metacrônicas, já que aproximada- 
mente $80 \%$ não são candidatos à operação curativa no momento do diagnóstico. As técnicas descritas abaixo são empregadas individualmente ou combinadas, com o objetivo de aumentar a ressecabilidade e, assim, oferecer possibilidade de cura ou ganho de sobrevida.

\section{Quimioterapia}

Com o aumento expressivo dos níveis de resposta aos modernos esquemas de quimioterapia, sua indicação ganha cada vez mais espaço como modalidade capaz de tornar ressecáveis lesões inicialmente irressecáveis (chamada de quimioterapia de conversão). A quimioterapia neoadjuvante permite também a observação do comportamento biológico da neoplasia, evitando cirurgias inúteis em pacientes com doenças extremamente agressivas, além de poder, ao menos teoricamente, fornecer um teste de quimiossensibilidade e auxiliar nas futuras estratégias terapêuticas. Os resultados atuais mostram que, uma vez apresentando resposta satisfatória, doenças inicialmente irressecáveis, adquirem após a hepatectomia, prognóstico comparável aos pacientes inicialmente ressecáveis ${ }^{8,9}$.

\section{dois tempos \\ Embolização portal e hepatectomia em}

Apesar dos avanços na cirurgia hepática, parênquima hepático funcional insuficiente após ressecção continua como uma das principais causas de irressecabilidade. Em condições normais, o risco de insuficiência hepática pós-operatória é elevado quando não se consegue preservar ao menos $25 \%$ do parênquima hepático funcional, aumentando de modo considerável a mortalidade pós-operatória. Alguns autores têm advogado que 30 a 40\% deva ser o limite de segurança nos pacientes submetidos à quimioterapia pré-operatória de longa duração ${ }^{39}$.

A embolização portal, descrita inicialmente por Makuuchi et al. ${ }^{40}$, visa induzir atrofia do hemifígado a ser ressecado e hipertrofia compensatória do fígado remanescente, aumentando tanto a ressecabilidade quanto a segurança da cirurgia. Em uma das séries publicadas, 63\% das MHCCR inicialmente irressecáveis, tornaram-se ressecáveis após embolização portal pré-operatória, com índice de sobrevida em cinco anos de $40 \%$, valor semeIhante ao da sobrevida dos pacientes inicialmente ressecáveis ${ }^{41}$.

Na tentativa de tirar o máximo proveito da embolização portal e limitar o risco de crescimento induzido das lesões localizadas no fígado contralateral ao segmento embolizado, Jaeck et al. ${ }^{42}$. descreveram modalidade bastante racional, chamada "hepatectomia em dois tempos associada à embolização portal" . Em um primeiro tempo, o fígado residual (geralmente um setor hepático ou um hemifígado) é completamente "limpo" de lesões através de ressecções e/ou técnicas ablativas. Segue-se com a embolização portal do hemifígado contralateral e consequente atrofia do fígado a ser ressecado e hipertrofia do futuro fígado remanescente. Espera-se cerca de quatro semanas para controle radiológico e, se houver crescimento adequado do remanescente hepático, indica-se uma segunda laparotomia para remover completamente as lesões metastáticas. A experiência com essa estratégia mostrou que ela pode ser realizada de maneira segura, com resultados, em cinco anos comparáveis aos pacientes portadores de MHCCR inicialmente ressecáveis ${ }^{43}$.

\section{Métodos ablativos}

Dentre os métodos ablativos, a experiência com a crioterapia é limitada e ablação com etanol é pouco efetiva no tratamento das MHCCR. A ablação por radiofrequência, por sua vez, é o método mais utilizado devido à sua simplicidade e efetividade como tratamento coadjuvante. Para o tratamento das MHCCR a radiofrequência pode ser utilizada de três maneiras distintas: a) como tratamento definitivo, quando a ressecção não pode ser indicada; b) como procedimento complementar à cirurgia; c) no tratamento das metástases recorrentes após ressecção.

Os resultados da utilização da radiofrequência no tratamento das MHCCR, mostram que esta deve ser utilizada em casos em que a maior lesão não supere $3 \mathrm{~cm}$ e quando existam no máximo três lesões ${ }^{44}$. Em estudo sobre a utilização de radiofrequência percutânea, o controle local foi obtido em $78 \%$ das lesões $<2,6 \mathrm{~cm}$, porém em apenas $47 \%$ das lesões entre $2,6-4,0 \mathrm{~cm}$ e em $32 \%$ das lesões $>4,0 \mathrm{~cm}^{45}$.

A localização anatômica da metástase é outra limitação adicional da radiofrequência (RF). Na proximidade de grandes vasos, por efeito de "roubo" de calor, o risco de ablação incompleta aumenta de maneira considerável. Na proximidade de estruturas biliares principais, como os ductos hepáticos direito e esquerdo, o risco de lesão térmica e estenose constitui contraindicação à sua utilização. A proximidade de órgãos extra-hepáticos também é apontada como fator de risco para complicações.

Devido às altas taxas de recidiva local e às limitações anatômicas descritas, a radiofrequência não está indicada em MHCCR ressecáveis. Uma meta-análise mostrou que a ressecção permanece o tratamento de escolha e que os resultados da RF são pouco superiores aos resultados dos tratamentos não cirúrgicos ${ }^{46}$.

Recomendações: O emprego de estratégias, com o intuito de buscar ressecções curativas em pacientes com doença inicialmente irressecável, é justificado pelas excelentes taxas de sobrevida apresentadas. A quimioterapia pré-operatória, com intuito de possibilitar ressecções curativas, deve ser empregada associada ou não aos anticorpos monoclonais, avaliando-se a resposta após três ou quatro ciclos e, assim que a ressecabilidade das lesões for constatada, o paciente deve ser operado. A embolização portal é técnica segura e comprovadamente eficiente, devendo ser empregada sempre que existir dúvi- 
da quanto à quantidade/qualidade do parênquima residual, especialmente em pacientes submetidos à múltiplas sessões de quimioterapia neoadjuvante, o limite de 30$40 \%$ de fígado residual deve ser respeitado. A radiofrequência permite o alargamento das indicações cirúrgicas com intuito curativo, em especial nos pacientes com múltiplas lesões bilobares.

\section{MHCCR SINCROONICAS}

As metástases sincrônicas ocorrem em 20 a 30\% dos pacientes, destes apenas 10 a $25 \%$ possuem lesões inicialmente ressecáveis. A conduta a ser tomada nesses casos, entretanto, ainda é controversa, em especial com relação ao momento ideal da ressecção hepática e da ressecção do tumor primário.

\section{a) Doença ressecável}

Os estudos iniciais dirigidos às ressecções simultâneas traziam resultados desanimadores com mortalidade e morbidade bastante superiores às ressecções separadas ou sequenciais ${ }^{47}$. Esses dados iniciais tornaram padrão, na maioria dos centros, a ressecção do tumor primário inicialmente e, num segundo tempo, a ressecção da doença mestastática.

Nos últimos anos, entretanto, devido ao número crescente de publicações mostrando bons resultados com as ressecções simultâneas, o paradigma do tratamento das MHCCR sincrônicas começou a mudar. Resultados comparáveis de sobrevida e morbidade perioperatória foram demonstrados independemente de ressecções realizadas simultaneamente ou estagiadas ${ }^{48}$ Os melhores candidatos à ressecção simultânea são os pacientes com tumores colônicos, devido a maior morbidade das ressecções retais, doença hepática limitada quanto ao número de lesões (geralmente menos do que três) e ao número de segmentos acometidos, buscando-se evitar ressecções maiores.

Alguns autores propuseram ainda uma terceira alternativa, o chamado "reversal approach" 49 . Nesta situação, a quimioterapia pré-operatória é seguida da ressecção hepática e, em um segundo tempo, o tumor primário é extirpado. A principal indicação desta estratégia reside em pacientes que apresentam doença hepática extensa e tumores primários assintomáticos em que o retardo no tratamento cirúrgico das metástases hepáticas pode torná-las em irressecáveis e comprometer sobremaneira a sobrevida dos pacientes.

De modo geral, as três estratégias apresentam resultados similares em termos de morbimortalidade e sobrevida global. Elas não devem ser vistas como excludentes, mas como complementares, e a escolha da sequência de tratamento deve ser feita individualmente, baseada nas condições clínicas do paciente e na extensão da doença tumoral.

\section{b) Doença irressecável}

A quimioterapia sistêmica é atualmente o principal tratamento para as MHCCR não ressecáveis. A incorporação de drogas, como a oxaliplatina e o irinotecan, conduziu a uma melhora significativa na taxa de resposta e sobrevida. O aparecimento das fluoropirimidinas orais, como a capecitabina, também trouxe novas opções ao tratamento destes pacientes.

A sobrevida mediana com quimioterapia exclusiva permanece em torno dos 20 meses e apenas 1 a $2 \%$ dos pacientes permanecem vivos após cinco anos ${ }^{50}$. No entanto, o aumento da eficácia dos modernos regimes quimioterápicos em promover regressão tumoral permite que 10 a $40 \%$ dos pacientes possam se converter em candidatos à ressecção ${ }^{8}$

Recomendações: Todo paciente com doença sincrônica deve ser avaliado quanto à possibilidade de ressecção sincrônica através de uma abordagem multidisciplinar. De forma resumida, a recomendação atual seria:

a) Ressecção do tumor primário sem dificuldade (em geral, tumores colônicos) e ressecção hepática menor (até três segmentos): ressecção sincrônica;

b) Ressecção do tumor primário sem dificuldade (em geral, tumores colônicos) e lesões hepáticas irressecáveis ou "limítrofes":

- quimioterapia $\rightarrow$ ressecção do tumor primário e primeiro tempo da hepatectomia ("limpar" lobo hepático com ou sem ligadura da veia porta contralateral) '! segundo tempo da hepatectomia (exérese completa das lesões hepáticas); ou

- "reversal approach" em casos selecionados (quimioterapia $\rightarrow$ hepatectomia $\rightarrow$ ressecção do tumor primário);

c) Ressecção complexa do tumor primário (por exemplo, tumores retais baixos) e ressecção hepática sem dificuldade: radio e quimioterapia neoadjuvante para o tumor primário $\rightarrow$ ressecção do tumor primário seguida de hepatectomia.

As ressecções sincrônicas só devem ser consideradas por equipes com experiência nas duas áreas.

\section{HEPATECTOMIA NA PRESENÇA DE DOENÇA EXTRA-HEPATICA}

A presença de doença extra-hepática já foi considerada contraindicação absoluta ao tratamento dos pacientes com MHCCR. Entretanto, no atual cenário de expansão das possibilidades de tratamento sistêmico e baixa morbimortalidade da terapia cirúrgica, a presença de doença extra-hepática não constitui por si só uma contraindicação absoluta à ressecção hepática, embora seja reconhecida como um dos principais fatores de mau prognóstico nesses pacientes. Carpizo et al. ${ }^{51}$ observaram sobrevida em cinco anos de 49\% em pacientes com MHCCR 
isoladas e $26 \%$ para pacientes com MHCCR e doença extra-hepática, sendo a recorrência da doença mais frequente nesse grupo. Os principais sítios de doença extra-hepática são os linfonodos do hilo hepático, pulmão e peritônio, sendo possível oferecer a grupos selecionados de pacientes a ressecção de toda doença tumoral (hepática e extrahepática), com resultados favoráveis.

O acometimento linfonodal do hilo hepático é uma disseminação da doença secundária presente no fígado, constituindo na realidade uma "metástase da metástase". Está presente em 3 a 33\% dos pacientes com MHCCR e é fator independente de menor sobrevida ${ }^{5}$. No entanto, o valor prognóstico desse acometimento linfonodal varia de acordo com a sua localização, podendo-se identificar subgrupos com melhor prognóstico. Em estudo prospectivo, Jaeck et al. ${ }^{52}$ separaram os linfonodos hilares em dois grupos que demonstraram significância prognóstica diferente, aqueles pacientes onde o comprometimento linfonodal se limitava ao ligamento hepatoduodenal e à porção retropancreática apresentaram sobrevida superior aos pacientes com comprometimento linfonodal junto à artéria hepática e tronco celíaco (sobrevida em três anos de 38\% vs 0\%). Outros autores confirmaram esses achados com sobrevida em cinco anos de até $25 \%$ em pacientes com acometimento do ligamento hepatogástrico e $0 \%$ em pacientes com acometimento do tronco celíaco ou para-aórtico ${ }^{51,53}$. Baseado nestes dados, pacientes selecionados com doença linfonodal restrita ao hilo hepático (sem acometimento do tronco celíaco) podem ser considerados candidatos à ressecção hepática.

Depois do fígado, o pulmão é a sede mais frequente de metástases de CCR,-cerca de $20 \%$ dos pacientes, sendo que 8 a $10 \%$ desenvolverão metástases pulmonares após a ressecção do tumor primário ${ }^{54}$. Os estudos existentes mostram benefício na ressecção das metástases pulmonares com sobrevida em cinco anos que varia de 27 a $74 \%{ }^{51}$. Os candidatos à ressecção são aqueles com poucas lesões pulmonares, ausência de linfonodos mediastinais acometidos e possibilidade de controle cirúrgico da doença hepática e pulmonar.
A doença peritoneal pode ocorrer em 3 a $28 \%$ dos pacientes com CCR e é a de tratamento mais controverso. O racional de sua abordagem baseia-se no conceito proposto segundo os quais as metástases peritoneais seriam uma forma regional da doença, oriunda da disseminação transmural de células tumorais e não causada pela sua disseminação sistêmica ${ }^{51}$. Apesar de resultados favoráveis à ressecção publicados, não existe ainda embasamento para seu uso fora de protocolos, em centros de referência.

Recomendações: Os principais sítios de doença extra-hepática são os linfonodos do hilo hepático, pulmão e peritônio. A presença de doença extra-hepática não deve ser considerada contraindicação absoluta para a ressecção hepática com intuito curativo. No entanto, os candidatos à terapêutica cirúrgica devem ser aqueles em que toda doença mensurável possa ser ressecada completamente, sem acréscimo importante na morbimortalidade, após avaliação multidisciplinar por grupos especializados.

\section{QUIMIOTERAPIA ADJUVANTE}

Apesar do importante acréscimo na sobrevida e possibilidade de cura obtida com a realização da ressecção completa das lesões hepáticas, cerca de 50 a 70\% dos pacientes apresentarão recidiva da doença no pós-operatório $^{35}$. Por essa razão, a utilização de quimioterapia perioperatória capaz de controle sistêmico da doença reveste de grande interesse nos portadores de MHCCR. Os dados existentes indicam benefício do uso da quimioterapia com 5-fluoracil e oxaliplatina no pós-operatório de pacientes submetidos à ressecção hepática por MHCCR em termos de sobrevida livre de doença e sobrevida global em cinco anos $15,16,69$.

Recomendações: O uso de quimioterapia sistêmica adjuvante associada à ressecção hepática fica preconizado amplamente, salvo na existência de contraindicação para a sua realização.

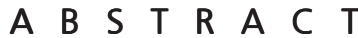

Approximately half of patients with colorectal cancer present with liver metastases during the course of their disease, which directly affect prognosis and is responsible for two thirds of deaths related to the disease. In the last two decades the treatment of liver metastases from colorectal cancer (CRCLM) provided significant gain in survival when all treatment options are available to the patient. In this context, surgical treatment remains as the only chance of cure, with five-year survival rates of 25-58\%. However, only $1 / 4$ of the patients have resectable disease at diagnosis. For this reason, one of the key points in the current management of patients with CRCLM is the development of strategies that facilitate complete resection of liver lesions. The advent and refinement of ablative methods have expanded the possibilities of surgical therapy. The emergence of new chemotherapy regimens and the introduction of targeted therapies has provided high response rates and has permanently altered the management of these patients. The multimodal therapy and the involvement of different medical specialties has increasingly enabled CRCLM treatment to approached the ideal treatment, i.e., an individualized one. Based on an extensive review of literature and on experience from some of the most important specialized centers of Brazil, the São Paulo Liver Club began a process of multi-institutional discussions that resulted in the recommendations that follow. These recommendations, however, are not intended to be absolute, but useful tools in the therapeutic decision process for this complex group of patients.

Key words: Neoplasms. Liver neoplasms. Colorectal neoplasms. Neoplasm metastasis. Therapeutics. 


\section{REFERÊNCIAS}

1. GLOBOCAN 2008. Section of cancer information, international agency for research on cancer. Lyon/France. Disponível em: http:/ /globocan.iarc.fr/factsheets/populations/ factsheet.asp? uno $=901 \mathrm{\# BOTH}$

2. Altekruse SF, Kosary CL, Krapcho M, Neyman N, Aminou R, Waldron W, et al. SEER Cancer Statistics Review, 1975-2007, National Cancer Institute. Disponível em: http://seer.cancer.gov/statfacts/ html/colorect.html

3. Brasil. Ministério da Saúde. Instituto Nacional do Câncer. Incidência de câncer no Brasil: Estimativa 2010. Rio de Janeiro, 2009. Disponível em: http://www.inca.gov.br/estimativa/2010/ estimativa20091201.pdf

4. Zavadsky KE, Lee YT. Liver metastases from colorectal carcinoma: incidence, resectability, and survival results. Am Surg. 1994;60(12):929-33

5. Abdalla EK, Adam R, Bilchik AJ, Jaeck D, Vauthey JN, Mahvi D. Improving resectability of hepatic colorectal metastases: expert consensus statement. Ann Surg Oncol. 2006;13(10):1271-80.

6. Adam R. Colorectal cancer with synchronous liver metastases. $\mathrm{Br}$ J Surg. 2007;94(2):129-31.

7. Fong, Y; Fortner J, Sun RL, Brennan MF, Blumgart LH. Clinical score for predicting recurrence after hepatic resection for metastatic colorectal cancer: analysis of 1001 consecutive cases. Ann Surg. 2000;230(3):309-18.

8. Adam R, Delvart V, Pascal G, Valeanu A, Castaing D, Azoulay D, et al. Rescue surgery for unresectable colorectal liver metastases downstaged by chemotherapy: a model to predict long-term survival. Ann Surg. 2004;240(4):644-57

9. Nordlinger $B$, Van Cutsem $E$, Rougier $P$, Köhne $C H$, Ychou $M$, Sobrero $A$, et al. Does chemotherapy prior to liver resection increase the potential for cure in patients with metastatic colorectal cancer? A report from the European Colorectal Metastases Treatment Group. Eur J Cancer. 2007;43(14):2037-45.

10. Tol J, Punt CJ. Monoclonal antibodies in the treatment of metastatic colorectal cancer: a review. Clin Ther. 2010;32(3):437-53.

11. Mitry E, Fields AL, Bleiberg H, Labianca R, Portier G, Tu D, et al. Adjuvant chemotherapy after potentially curative resection of metastases from colorectal cancer: a pooled analysis of two randomized trials. J Clin Oncol. 2008;26(30):4906-11.

12. Desch CE, Benson AB 3rd, Somerfield MR, Flynn PJ, Krause C, Loprinzi $\mathrm{CL}$, et al. Colorectal cancer surveillance: 2005 update of an American Society of Clinical Oncology practice guideline. J Clin Oncol. 2005;23(33):8512-9.

13. Tan E, Gouvas N, Nicholls RJ, Ziprin P, Xynos E, Tekkis PP. Diagnostic precision of carcinoembryonic antigen in the detection of recurrence of colorectal cancer. Surg Oncol. 2009;18(1):15-24.

14. Blyth S, Blakeborough A, Peterson M, Cameron IC, Majeed AW. Sensitivity of magnetic resonance imaging in the detection of colorectal liver metastases. Ann R Coll Surg Engl. 2008;90(1):25-8.

15. Maithel SK, Ginsberg MS, D'Amico F, DeMatteo RP, Allen PJ, Fong $Y$, et al. Natural history of patients with subcentimeter pulmonary nodules undergoing hepatic resection for metastatic colorectal cancer. J Am Coll Surg. 2010;210(1):31-8

16. Rappeport ED, Loft A, Berthelsen AK, von der Recke P, Larsen PN, Mogensen AM, et al. Contrast-enhanced FDG-PET/CT vs. SPIO enhanced MRI vs. FDG-PET vs. CT in patients with liver metastases from colorectal cancer: a prospective study with intraoperative confirmation. Acta Radiol. 2007;48(4):369-78.

17. Coenegrachts K, De Geeter F, ter Beek L, Walgraeve N, Bipat $S$, Stoker J, et al. Comparison of MRI (including SS SE-EPI and SPIOenhanced MRI) and FDG-PET/CT for the detection of colorectal liver metastases. Eur Radiol. 2009;19(2):370-9.

18. Strasberg SM, Dehdashti F. Role of FDG-PET staging in selecting the optimum patient for hepatic resection of metastatic colorectal cancer. J Surg Oncol. 2010;102(8):955-9.

19. Mazzoni G, Napoli A, Mandetta S, Miccini M, Cassini D, Gregori M, et al. Intra-operative ultrasound for detection of liver metastases from colorectal cancer. Liver Int. 2008;28(1):88-94.
20. Adam R, Bismuth H, Castaing D, Waechter F, Navarro F, Abascal A, et al. Repeat hepatectomy for colorectal liver metastases. Ann Surg. 1997;225(1):51-60.

21. Bhattacharjya S, Aggarwal R, Davidson BR. Intensive follow-up after liver resection for colorectal liver metastases: results of combined serial tumour marker estimations and computed tomography of the chest and abdomen - a prospective study. $\mathrm{Br}$ J Cancer. 2006;95(1):21-6.

22. Zacharias T, Jaeck D, Oussoultzoglou E, Bachellier P, Weber JC. First and repeat resection of colorectal liver metastases in elderly patients. Ann Surg. 2004;240(5):858-65.

23. Kokudo N, Miki Y, Sugai $S$, Yanagisawa A, Kato $Y$, Sakamoto $Y$ et al. Genetic and histological assessment of surgical margins in resected liver metastases from colorectal carcinoma: minimum surgical margins for successful resection. Arch Surg. 2002;137(7):833-40

24. Nordlinger B, Van Cutsem E, Gruenberger T, Glimelius B, Poston $G$, Rougier $P$, et al. Combination of surgery and chemotherapy and the role of targeted agents in the treatment of patients with colorectal liver metastases: recommendations from an expert panel. Ann Oncol. 2009;20(6):985-92.

25. Bockhorn $M$, Frilling $A$, Frühauf NR, Neuhaus J, Molmenti $E$, Trarbach $T$, et al. Survival of patients with synchronous and metachronous colorectal liver metastases-is there a difference? J Gastrointest Surg. 2008;12(8):1399-405.

26. Allen PJ, Kemeny N, Jarnagin W, DeMatteo R, Blumgart L, Fong $Y$. Importance of response to neoadjuvant chemotherapy in patients undergoing resection of synchronous colorectal liver metastases. J Gastrointest Surg. 2003;7(1):109-15.

27. Capussotti L, Vigano' L, Ferrero A, Lo Tesoriere R, Ribero D, Polastri R. Timing of resection of liver metastases synchronous to colorectal tumor: proposal of prognosis-based decisional model. Ann Surg Oncol. 2007;14(3):1143-50.

28. Nordlinger B, Sorbye H, Glimelius B, Poston GJ, Schlag PM, Rougier $P$, et al. Perioperative chemotherapy with FOLFOX4 and surgery versus surgery alone for resectable liver metastases from colorectal cancer (EORTC Intergroup trial 40983): a randomised controlled trial. Lancet. 2008;371(9617):1007-16

29. Chua TC, Saxena A, Liauw W, Kokandi A, Morris DL. Systematic review of randomized and nonrandomized trials of the clinical response and outcomes of neoadjuvant systemic chemotherapy for resectable colorectal liver metastases. Ann Surg Oncol. 2010; 17(2):492-501

30. Karoui M, Penna C, Amin-Hashem M, Mitry E, Benoist S, Franc B, et al. Influence of preoperative chemotherapy on the risk of major hepatectomy for colorectal liver metastases. Ann Surg 2006:243(1):1-7.

31. Aloia T, Sebagh M, Plasse M, Karam V, Lévi F, Giacchetti $S$, et al Liver histology and surgical outcomes after preoperative chemotherapy with fluorouracil plus oxaliplatin in colorectal cancer liver metastases. J Clin Oncol. 2006:24(31):4983-90.

32. Peppercorn PD, Reznek RH, Wilson P, Slevin ML, Gupta RK Demonstration of hepatic steatosis by computerized tomography in patients receiving 5-fluorouracil-based therapy for advanced colorectal cancer. Br J Cancer. 1998;77(11):2008-11.

33. Nordlinger B, Vauthey JN, Poston G, Benoist S, Rougier P, Van Cutsem $\mathrm{E}$. The timing of chemotherapy and surgery for the treatment of colorectal liver metastases. Clin Colorectal Cancer. 2010;9(4):212-8

34. Takamoto T, Hashimoto T, Sano K, Maruyama Y, Inoue K, Ogata $S$, et al. Recovery of liver function after the cessation of preoperative chemotherapy for colorectal liver metastasis. Ann Surg Oncol. 2010;17(10:2747-55

35. Kesmodel SB, Ellis LM, Lin E, Chang GJ, Abdalla EK, Kopetz S, et al. Preoperative bevacizumab does not significantly increase postoperative complication rates in patients undergoing hepatic surgery for colorectal cancer liver metastases. J Clin Oncol. 2008;26(32):5254-60.

36. Wieser M, Sauerland S, Arnold D, Schmiegel W, Reinacher-Schick A. Peri-operative chemotherapy for the treatment of resectable 
liver metastases from colorectal cancer: A systematic review and meta-analysis of randomized trials. BMC Cancer. 2010;10:309.

37. Adam R, Bhangui P, Poston G, Mirza D, Nuzzo G, Barroso E, et al. Is perioperative chemotherapy useful for solitary, metachronous, colorectal liver metastases? Ann Surg. 2010;252(5):774-87.

38. Lalmahomed ZS, Ayez N, van der Pool AE, Verheij J, ljzermans JN, Verhoef C. Anatomical versus nonanatomical resection of colorectal liver metastases: is there a difference in surgical and oncological outcome? World J Surg. 2011:35(3):656-61.

39. Adam R, Lucidi V, Bismuth H. Hepatic colorectal metástases: methods of improving resecability. Surg Clin North Am. 2004;84(2):659-71.

40. Makuuchi M, Thai BL, Takayasu K, Takayama T, Kosuge T, Gunvén $P$, et al. Preoperative portal embolization to increase safety of major hepatectomy for hilar bile duct carcinoma: a preliminary report. Surgery. 1990;107(5):521-7.

41. Azoulay D, Castaing D, Smail A, Adam R, Cailliez V, Laurent A, et al. Resection of nonresectable liver metástases from colorectal cancer after percutaneous portal vein embolization. Ann Surg. 2000;231(4):480-6.

42. Jaeck D, Oussoultzoglou E, Rosso E, Greget M, Weber JC, Bachellier P. A two-stage hepatectomy procedure combined with portal vein embolization to achieve curative resection for initially unresectable multiple bilobar colorectal liver metastases. Ann Surg. 2004;240)6):1037-49.

43. Wicherts DA, Miller R, de Haas RJ, Bitsakou G, Vibert E, Veilhan LA, et al. Long-term results for two-stage hepatectomy for irressectable colorectal cancer liver metastases. Ann Surg. 2008;248(6):994-1005

44. Berber E, Pelley R, Siperstein AE. Predictors of survival after radiofrequency thermal ablation of colorectal cancer metastases to the liver: a prospective study. J Clin Oncol. 2005;23(7):1358-64.

45. Solbiati L, Livraghi T, Goldberg SN, lerace T, Meloni F, Dellanoce $M$, et al. Percutaneous radio-frequency ablation of hepatic metastases form colorectal cancer: long-term results in 117 patients. Radiology. 2001;221(1):159-66.

46. Mulier S, Ni Y, Jamart J, Ruers T, Marchal G, Michel L. Local recurrence after hepatic radiofrequency coagulation: multivariate meta-analysis and review of contributing factors. Ann Surg. 2005;242(2):158-71.

47. Nordlinger B, Guiguet M, Vaillant JC, Balladur P, Boudjema K, Bachellier $\mathrm{P}$, et al. Surgical resection of colorectal carcinoma metastases to the liver. A prognostic scoring system to improve case selection, based on 1568 patients. Association Française de Chirurgie. Cancer. 1996;77(7):1254-62.
48. Martin RC 2nd, Augenstein V, Reuter NP, Scoggins CR, McMasters KM. Simultaneous versus staged resection for synchronous colorectal cancer liver metastases. J Am Coll Surg. 2009;208(5):84250.

49. Mentha G, Majno PE, Andres A, Rubbia-Brandt L, Morel P, Roth $A D$. Neoadjuvant chemotherapy and resection of advanced synchronous liver metastases before treatment of the colorectal primary. Br J Surg. 2006;93(7):872-8.

50. Hobday TJ, Kugler JW, Mahoney MR, Sargent DJ, Sloan JA, Fitch $T R$, et al. Efficacy and quality-of-life data are related in a phase II trial of oral chemotherapy in previously untreated patients with metastatic colorectal carcinoma. J Clin Oncol. 2002;20(23):457480.

51. Carpizo DR, Are C, Jarnagin W, Dematteo R, Fong $Y$, Gönen $M$, et al. Liver resection for metastatic colorectal cancer in patients with concurrent extrahepatic disease: results in 127 patients treated at a single center. Ann Surg Oncol. 2009;16(8):2138-46.

52. Jaeck D, Nakano H, Bachellier P, Inoue K, Weber JC, Oussoultzoglou $\mathrm{E}$, et al. Significance of hepatic pedicle lymph node involvement in patients with colorectal liver metastases: a prospective study. Ann Surg Oncol. 2002;9(5):430-8.

53. Adam R, de Haas RJ, Wicherts DA, Aloia TA, Delvart V, Azoulay D, et al. Is hepatic resection justified after chemotherapy in patients with colorectal liver metastases and lymph node involvement? J Clin Oncol. 2008;26(22):3672-80.

54. Shah SA, Haddad R, Al-Sukhni W, Kim RD, Greig PD, Grant DR, et al. Surgical resection of hepatic and pulmonary metastases from colorectal carcinoma. J Am Coll Surg. 2006;202(3):468-75.

Recebido em 01/07/2012

Aceito para publicação em 01/10/2012

Conflito de interesse: nenhum

Fonte de financiamento: nenhuma

\section{Como citar este artigo:}

Lupinacci RM, Coelho FF, Perini MV, Lobo EJ, Ferreira FG, Szutan LA, Lopes GJ, Herman P; Clube do Fígado de São Paulo. Manejo atual das metástases hepáticas de câncer colorretal. Recomendações do Clube do Fígado de São Paulo. Rev Col Bras Cir. [periódico na Internet] 2013;40(3). Disponível em URL: http://www.scielo.br/rcbc

\section{Endereço para correspondência:}

Paulo Herman

E-mail:pherman@uol.com.br 